\title{
关于函数类 $\Omega_{P}^{r+1}[0,1]$ 的宽度估计 \\ 孙永生黄达人 \\ (北京师氾大学数学系) (浙江大学数学系,杭州)
}

\section{§1. 引言}

设 $l \geqslant 1, r=2 l, t_{1}, \cdots, t_{l} \geqslant 0, D=-\frac{d}{d x}, I$ 为恒等算子, 记

$$
Q_{r+1}(D)=D \prod_{j=1}^{l}\left(D^{2}-t_{j}^{2} I\right), \quad q_{r}(x)=\prod_{j=1}^{l}\left(x^{2}-t_{j}^{2}\right),
$$

$Q_{p}^{++1}[0,1]=\left\{f(x) ; f^{(r)}\right.$ 在 $[0,1]$ 上绝对连续, 且

$$
\left.\left\|Q_{r+1}(D) f(\cdot)\right\|_{p} \leqslant 1, f^{(2 k-1)}(0)=f^{(2 k-1)}(1)=0, k=1, \cdots, l\right\},
$$

于是, $f \in Q_{p}^{r+1}[0,1]$, 当且仅当

其中 $c \in R,\|g\|_{p} \leqslant 1$. 而

$$
f(x)=c+\int_{0}^{1} K(x, y) g(y) d y \equiv c+K g,
$$

$$
K(x, y)=-2 \sum_{k=1}^{\infty} \frac{\cos k \pi x \sin k \pi y}{k \pi q_{r}(i k \pi)} .
$$

又记

$$
d_{n}\left[Q_{p}^{r+1} ; L_{s}\right]=\inf _{M_{n}} \sup _{f \in Q_{p}^{+1}} \min _{\varphi \in M_{n}}\|f-\varphi\|_{s},
$$

这里 $M_{n} \subset L_{s}[0,1]$ 是 $L_{s}$ 内任取的 $n$ 维子空间, 该量称为函数类 $Q_{p}^{+1}$ 在 $L_{s}$ 内的 Kolmogorov 宽度.

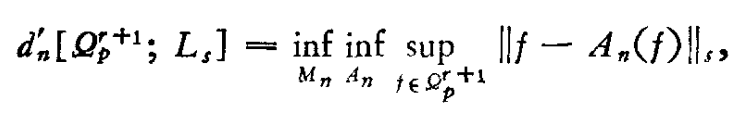

这里 $M_{n} \subset L_{s}[0,1]$ 为任一 $n$ 维子空间, 而 $A_{n}(f)$ 是 $Q_{p}^{+1} \rightarrow M_{n}$ 的任一线性连续映射, 该 量称为 $Q_{p}^{++1}$ 在 $L_{s}$ 内的 $n$ 维线性宽度. 由于

$$
K(x, y)=\sum_{k=1}^{\infty} \frac{\sin k \pi(x-y)}{k \pi q_{r}(i k \pi)}-\sum_{k=1}^{\infty} \frac{\sin k \pi(x+y)}{k \pi q_{r}(i k \pi)},
$$

那么对 $f=c+K g \in Q_{p}^{r+1}$, 有

$$
f(x)=c+\int_{-1}^{1} \sum_{k=1}^{\infty} \frac{\sin k \pi(x-y)}{k \pi q_{r}\left(i k_{x}\right)} \tilde{g}(y) d y,
$$

这里 $\tilde{g}(y)$ 是 $g(y)$ 的以 2 为周期的奇延拓, 在 (1.6)式内令 $x$ 取 $[0,1]$ 以外的值, 便得到 $f(x)$ 以 2 为周期的偶延拓 $\hat{f}(x)$. 记

$$
\widetilde{K}_{r+1}(x)=\sum_{k=1}^{\infty} \frac{\sin k \pi x}{k \pi q_{r}(i k x)},
$$

本文 1983 年 2 月 10 日收到. 
则对 $f=c+K g \in Q_{p}^{+1}$ 有

\section{§2. 一个极值问题}

$$
\hat{f}=c+\widetilde{K}_{r+1} * \tilde{g} .
$$

若记

$$
\begin{gathered}
\Lambda_{n}=\left\{\xi: \xi=\left(\xi_{1}, \cdots, \xi_{n}\right), 0=\xi_{0}<\xi_{1}<\cdots<\xi_{n}<\xi_{n+1}=1\right\}, \\
\Gamma_{n}=\left\{h_{\xi}(t): \xi \in \Lambda_{m},(m \leqslant n), h_{\xi}(t)=(-1)^{i}, \xi_{j} \leqslant t<\xi_{j+1}, j=0, \cdots, m\right\},
\end{gathered}
$$

考虑如下的极小问题:

$$
\min _{c \in R, h_{\xi} \in \Gamma_{n}}\left\|_{c}+K h_{\xi}\right\|_{p}, \quad(1<p<\infty),
$$

存在 $c^{*} \in R, \xi^{*}=\left\{0=\xi_{0}^{*}<\xi_{1}^{*}<\cdots<\xi_{m}^{*}<\xi_{m+1}^{*}=1\right\} \in \Lambda_{m},(m \leqslant n)$, 使成立

记

$$
\min _{c \in R, h_{\xi} \in \Gamma_{n}}\left\|c+K h_{\xi}\right\|_{p}=\left\|c^{*}+K h_{\xi^{*}}\right\|_{p} .
$$

$$
P_{\xi^{*}}(x)=c^{*}+\int_{0}^{1} K(x, y) h_{\xi^{*}}(y) d y,
$$

应用和文献 $[1]$ 内一样的分析可得

$$
\begin{gathered}
\int_{0}^{1}\left|P_{\xi^{*}}(x)\right|^{p-1} \operatorname{sgn}\left(P_{\xi^{*}}(x)\right) d x=0, \\
\int_{0}^{1}\left|P_{\xi^{*}}(x)\right|^{p-1} \operatorname{sgn}\left(P_{\xi^{*}}(x)\right) K\left(x, \xi_{i}^{*}\right) d x=0, j=1, \cdots, m .
\end{gathered}
$$

令

$$
G(y)=\int_{0}^{1}\left|P_{\xi^{*}}(x)\right|^{p-1} \operatorname{sgn}\left(P_{\xi^{*}}(x)\right) K(x, y) d x,
$$

则

$$
G\left(\xi_{j}^{*}\right)=0, j=0,1, \cdots, m+1 .
$$

引理 $2.1 G(y)$ 在 $(0,1)$ 内除 $\xi_{i}^{*}$ 外别无零点, 且每一 $\xi_{i}^{*}(j=1, \cdots, m)$ 都是 $G(y)$ 的变号点.

证令

$$
\begin{aligned}
& \hat{P}_{\xi^{*}}(x)=c^{*}+\int_{-1}^{1} \widetilde{K}_{r+1}(x-y) \tilde{h}_{\xi^{*}}(y) d y, \\
& \widetilde{G}(y)=\int_{-1}^{1}\left|\hat{P}_{\xi^{*}}(x)\right|^{p-1} \operatorname{sgn}\left(\hat{P}_{\xi^{*}}(x)\right) \widetilde{K}_{r+1}(x-y) d x,
\end{aligned}
$$

$\hat{P}_{\xi^{*}}(x), \widetilde{G}(y)$ 各是 $P_{\xi^{*}}(x), G(y)$ 的以 2 为周期的偶延拓和奇延拓, 对 $\widetilde{G}(y)$ 成立着

$$
\widetilde{G}\left( \pm \xi_{i}^{*}\right)=0, \quad j=0,1, \cdots, m+1 .
$$

假定 $G(y)$ 在 $(0,1)$ 内的零点个数大于 $m$ (重根要计算它的重复次数), 则 $\widetilde{G}(y)$ 在 $(-1,1)$ 内的零点个数大于 $2 m+3$, 把 \pm 1 计算在内, 根据 Rolle 定理得知 $\tilde{G}^{\prime}(y)$ 在 $(-1,1)$ 内零 点个数大于 $2 m+4$. 注意到

$$
\begin{aligned}
& \tilde{G}^{\prime}(y)=\int_{-1}^{1}\left|\hat{P}_{\xi^{*}}(x)\right|^{p-1} \operatorname{sgn}\left(\hat{P}_{\xi^{*}}(x)\right) K_{r}(x-y) d y, \\
& \left(D^{2}-t_{l}^{2} l\right) \widetilde{G}^{\prime}(y)=\int_{-1}^{1}\left|\hat{P}_{\xi^{*}}(x)\right|^{p-1} \operatorname{sgn}\left(\hat{P}_{\xi^{*}}(x)\right) K_{r-2}(x-y) d x, \\
& \ldots \ldots . \\
& q_{r}(D) \tilde{G}^{\prime}(y)=\left|\hat{P}_{\xi^{*}}(y)\right|^{p-1} \operatorname{sgn}\left(\hat{P}_{\xi^{*}}(y)\right),
\end{aligned}
$$

第 12 期 
此处

$$
\left\{\begin{aligned}
K_{r}(x) & =\sum_{k=1}^{\infty} \frac{\cos k \pi x}{q_{r}(i k \pi)}, \\
K_{r-2}(x) & =\sum_{k=1}^{\infty} \frac{\cos k \pi x}{q_{2 l-2}(i k \pi)}, \quad q_{2 l-2}(x)=\sum_{i=1}^{l-1}\left(x^{2}-t_{l}^{2}\right),
\end{aligned}\right.
$$

$P_{\xi^{*}}(x)$ 在 $[-1,1)$ 内至少有 $2 m+4$ 个零点, 但由于

$$
\hat{P}_{\xi^{*}}(x)=c^{*}+\int_{-1}^{1} K_{r}(x-t) \int_{-1}^{1} D_{1}(t-y) \hat{h}_{\xi^{*}}(y) d y d t,
$$

此处

$$
D_{1}(t)=\sum_{k=1}^{\infty} \frac{\sin k \pi t}{k \pi}
$$

而函数 $\int_{-1}^{1} D_{1}(t-y) \hat{h}_{\xi}^{*}(y) d y$ 是以 2 为周期的偶函数, 它在每一区间 $\left[\xi_{z-1}^{*}, \xi_{z}^{*}\right]$ 上是一次 函数,故其在一个周期内零点的个数小于或等于 $2(m+1)$. 从而 $\hat{P}_{\xi} *(x)$ 在一周期内的零点 个数不大于 $2(m+1)$. 得到矛盾. 引理 2.1 证完.

$$
\Delta_{k}=\left[\xi_{k-1}^{*}, \xi_{k}^{*}\right], k=-m, \cdots,-1,0, \cdots, m+1 .\left|\Delta_{k}\right|=\xi_{k}^{*}-\xi_{k-1}^{*}, \delta=\min \left\{\left|\Delta_{k}\right|\right\} .
$$

置 $\widetilde{H}(x)=\widetilde{G}(x)+\widetilde{G}(x+\delta)$, 可以证得

引理 $2.2\left|\Delta_{-m}\right|=\cdots=\left|\Delta_{m+1}\right|=\frac{1}{m+1}$. 还有

引理 $2.3 \quad c^{*}=0$.

证 由引理 2.2 得

$$
P_{\xi^{*}}(x)=\int_{0}^{1} K(x, y) \operatorname{sgn}(\sin (m+1) \pi y) d y+c^{*},
$$

注意

$$
\begin{aligned}
& \int_{0}^{1} K(x, y) \operatorname{sgn}(\sin (m+1) \pi y) d y \\
= & \frac{4}{\pi(m+1)} \sum_{k=0}^{\infty} \frac{\cos (2 k+1)(m+1) \pi x}{(2 k+1)^{2} q_{r}(i(2 k+1)(m+1) \pi)},
\end{aligned}
$$

以及

$$
\operatorname{sgn} \int_{0}^{1} K(x, y) \operatorname{sgn}(\sin (m+1) \pi y) d y=\operatorname{sgn}(\cos (m+1) \pi x),
$$

还有(2.6)式, 得到

$$
\min _{\alpha}\left\|P_{\xi^{*}}(x)-\alpha\right\|_{p}=\left\|P_{\xi^{*}}(x)\right\|_{p}
$$

但通过直接验算易得

$$
\int_{0}^{1}\left|\int_{0}^{1} K(x, y) \operatorname{sgn}(\sin (m+1) \pi y) d y\right|^{p-1} \operatorname{sgn}(\cos (m+1) \pi x) d x=0,
$$

根据 $L_{p}(1<p<\infty)$ 尺度下最佳逼近元的唯一性得

$$
\left\|P_{\xi} *(x)\right\|_{p}=\left\|\int_{0}^{1} K(x, y) \operatorname{sgn}(\sin (m+1) \pi y) d y\right\|_{p} .
$$

由此得到 $c^{*}=0$.

引理 $2.4 m-n$. 
证 引人函数

$$
G_{j+1, r}(x)=\frac{4}{\pi(j+1)} \sum_{k=0}^{\infty} \frac{\cos (2 k+1) \pi x}{(2 k+1)^{2} q_{r}(i(2 k+1)(j+1) \pi)},
$$

仿炤文献[2]可得当 $j^{\prime}>i$ 时有

$$
\left.\left|G_{i^{\prime}+1, r}(x)\right|<\left|G_{i+1, r}(x)\right| . \forall x \& \mid 0, \frac{1}{2}\right) \cup\left(\frac{1}{2}, 1\right] .
$$

由此即可推知 $m=n$.

由引理 2.1-2.4 得: 对 $1<p<\infty$, 有

$$
\min _{c \in R, h_{\xi} \in \Gamma_{n}}\left\|c+K h_{\xi}\right\|_{p}=\left\|\int_{0}^{1} K(\cdot, y) \operatorname{sgn}(\sin (n+1) \pi y) d y\right\|_{p},
$$

再令 $p \rightarrow 1^{+}, p \rightarrow+\infty$, 便得

定理 1 对 $1 \leqslant p \leqslant \infty$, 有

$$
\min _{c \in R, h_{\xi} \in \Gamma_{n}}\left\|c+K h_{\xi}\right\|_{p}=\left\|\int_{0}^{1} K(\cdot, y) \operatorname{sgn}(\sin (n+1) \pi y) d y\right\|_{p} .
$$

\section{§3. 对偶的极值问题}

设 $1 \leqslant p \leqslant \infty, \frac{1}{p}+\frac{1}{p^{\prime}}=1$. 记

$$
\Gamma_{n}^{\prime}=\left\{h_{\xi}: h_{\xi} \in \Gamma_{n}, \text { 且 } \int_{0}^{1} h_{\xi}(x) d x=0\right\} .
$$

考虑上节的对偶极小问题:

类似于 $\$ 2$ 可得

$$
\min _{h_{\xi} \in \Gamma_{n}^{\prime}} \|\left.\int_{0}^{1} K(y, x) h_{\xi}(y) d y\right|_{p^{\prime}}
$$

定理 2 对 $1 \leqslant p \leqslant \infty$. 有

$$
\min _{h_{\xi} \in \Gamma_{n}^{\prime}}\left\|\int_{0}^{1} K(y, x) h_{\xi}(y) d y\right\|_{p^{\prime}}=\left\|\int_{0}^{1} K(y, \cdot) \operatorname{sgn}(\cos n \pi y) d y\right\|_{p^{\prime}},
$$

且由直接计算知

$$
\int_{0}^{1} K\left(y, \frac{i}{n}\right) \operatorname{sgn}(\cos n \pi y) d y=0, i=0,1, \cdots, n .
$$

\section{$\S 4 . \Omega_{p}^{+1}[0,1]$ 类一些宽度的计算}

\section{定理 3}

而对 $n \geqslant 1$, 有

$$
d_{0}\left[\Omega_{\infty}^{r+1}, L_{s}\right]=d_{0}\left[\Omega_{p}^{r+1}, L_{1}\right]=\infty
$$

$$
\begin{aligned}
& d_{n}^{\prime}\left[Q_{\infty}^{r+1}, L_{s}\right]=d_{n}\left[Q_{\infty}^{+1}, L_{s}\right]=\left\|\int_{0}^{1} K(\cdot, y) \operatorname{sgn}(\sin n \pi y) d y\right\|_{s} ; \\
& d_{n}^{\prime}\left[Q_{p}^{r+1}, L_{1}\right]=d_{n}\left[Q_{p}^{r+1}, L_{1}\right]=\left\|\int_{0}^{1} K(y, \cdot) \operatorname{sgn}(\cos n \pi y) d y\right\|_{p^{\prime}}
\end{aligned}
$$

证 (4.1)式根据宽度的一般性质立即可得. 当 $n \geqslant 1$ 时,据文献[ 1 ]的一般定理有

$$
\begin{aligned}
& d_{n}\left[Q_{\infty}^{r+1}, L_{s}\right] \geqslant \min _{c \in R, h_{\xi} \in \Gamma_{n-1}}\left\|c+K h_{\xi}\right\|_{s} \\
= & \left\|\int_{0}^{1} K(\cdot, y) \operatorname{sgn}(\sin n \pi y) d y\right\|_{s} .
\end{aligned}
$$


为得到 $d_{n}\left[Q_{\infty}^{r+1}, L_{s}\right]$ 的上界估计,考虑 $n$ 维子空间

$$
U_{n}=\operatorname{span}\left\{1, K\left(x, \frac{1}{n}\right), \cdots, K\left(x, \frac{n-1}{n}\right)\right\},
$$

对 $f \in c+K g \in Q_{\infty}^{++1}[0,1]$, 以 $S_{f} \in U_{n}$ 表示 $f$ 在 $\left\{\frac{2 i-1}{2 n}\right\}_{i=1}^{n}$ 的插值样条, 即

$$
S_{f}\left(\frac{2 i-1}{2 n}\right)=f\left(\frac{2 i-1}{2 n}\right), \quad i=1, \cdots, n .
$$

由文献[3], 这样的 $S_{f}$ 是唯一存在的,且有关系式

$$
f(x)-S_{f}(x)=\int_{0}^{1} G(x, y) g(y) d y,
$$

其中 $G(x, y)=K(x, y)-S_{K}(x, y)$, 而 $S_{K}(x, y) \in U_{n}$ 表示函数 $K(x, y)$ 的以 $x$ 为自变 量的插值样条. 不难得到

$$
G(x, y)=(-1)^{l+1} \operatorname{sgn}(\sin n \pi y) \cdot \operatorname{sgn}(\cos n \pi x)|G(x, y)|,
$$

这里的 $l$ 就是 (1.1) 式的 $l$. 于是对 $f \in Q_{\infty}^{+1}[0,1]$, 成立

$$
\left|f-S_{f}\right| \leqslant \int_{0}^{1}|G(x, y)| d y=\left|\int_{0}^{1} G(x, y) \operatorname{sgn}(\sin n \pi y) d y\right|,
$$

若特别取 $f_{0}(x)=\int_{0}^{1} K(x, y) \operatorname{sgn}(\sin n \pi y) d y$, 则由于 $f_{0}\left(\frac{2 i-1}{2 n}\right)=0, i=1, \cdots, n$, 所以 $f_{0}(x)$ 的插值样条 $S_{f_{0}}(x) \equiv 0$, 于是成立

$$
\begin{aligned}
& \int_{0}^{1} K(x, y) \operatorname{sgn}(\sin n \pi y) d y=f_{0}(x)=f_{0}(x)-S_{f_{i}}(x) \\
& =\int_{0}^{1} G(x, y) \operatorname{sgn}(\sin n \pi y) d y,
\end{aligned}
$$

由(4.6)式得

$$
\left\|f-S_{f}\right\| \leqslant\left\|f_{0}(x)\right\|=\left\|\int_{0}^{1} K(\cdot, y) \operatorname{sgn}(\sin n \pi y) d y\right\|_{s},
$$

于是

$$
d_{n}\left[\Omega_{\infty}^{+1}, L_{s}\right] \leqslant d_{n}^{\prime}\left[Q_{\infty}^{+1}, L_{s}\right] \leqslant\left\|\int_{0}^{1} K(\cdot, y) \operatorname{sgn}(\sin n \pi y) d y\right\|_{s}
$$

结合(4.4)式即得(4.2)式. (4.3)式类似地可以证得.

\section{参考文献}

[1] Micchelli, C. A., J. Appro. Theory, 24 (1978), 51-77.

[2] 孙永生,中国科学, A 辑, 1983, 8: 677-688.

[3] 黄达人,王建忠,数学年刊，4(1983)，3: 349-360. 